\title{
Stochastic properties of the frequency dynamics in real and synthetic power grids
}

\author{
Mehrnaz Anvari $\odot,{ }^{1,2,{ }^{*}}$ Leonardo Rydin Gorjão $\odot,,^{3,4, \dagger}$ Marc Timme, ${ }^{5,6, \ddagger}$ Dirk Witthaut $\odot,{ }^{3,4, \S}$ \\ Benjamin Schäfer ${ }^{0},{ }^{7,5,6, \|}$ and Holger Kantz ${ }^{1}$ \\ ${ }^{1}$ Max Planck Institute for the Physics of Complex Systems (MPIPKS), 01187 Dresden, Germany \\ ${ }^{2}$ Potsdam Institute for Climate Impact Research (PIK), Member of the Leibniz Association, P.O. Box 6012 03, D-14412 Potsdam, Germany \\ ${ }^{3}$ Forschungszentrum Jülich, Institute for Energy and Climate Research - Systems Analysis and Technology Evaluation (IEK-STE), \\ 52428 Jülich, Germany \\ ${ }^{4}$ Institute for Theoretical Physics, University of Cologne, 50937 Köln, Germany \\ ${ }^{5}$ Chair for Network Dynamics, Center for Advancing Electronics Dresden (cfaed) and Institute for Theoretical Physics, \\ Technical University of Dresden, 01062 Dresden, Germany \\ ${ }^{6}$ Network Dynamics, Max Planck Institute for Dynamics and Self-Organization (MPIDS), 37077 Göttingen, Germany \\ ${ }^{7}$ School of Mathematical Sciences, Queen Mary University of London, London E1 4NS, United Kingdom
}

(Received 1 October 2019; accepted 19 December 2019; published 19 March 2020)

\begin{abstract}
The frequency constitutes a key state variable of electrical power grids. However, as the frequency is subject to several sources of fluctuations, ranging from renewable volatility to demand fluctuations and dispatch, it is strongly dynamic. Yet, the statistical and stochastic properties of the frequency fluctuation dynamics are far from fully understood. Here we analyze properties of power-grid frequency trajectories recorded from different synchronous regions. We highlight the non-Gaussian and still approximately Markovian nature of the frequency statistics. Furthermore, we find that the frequency displays significant fluctuations exactly at the time intervals of regulation and trading, confirming the need of having a regulatory and market design that respects the technical and dynamical constraints in future highly renewable power grids. Finally, employing a recently proposed synthetic model for the frequency dynamics, we combine our statistical and stochastic analysis and analyze in how far dynamically modeled frequency properties match the ones of real trajectories.
\end{abstract}

DOI: 10.1103/PhysRevResearch.2.013339

\section{INTRODUCTION}

A stable electric power supply is essential for the functioning of our society [1]. The ongoing energy transition towards renewable generation fundamentally changes the conditions for the operation of the power system [2]. A better understanding of the dynamics, control, and variability of this highly complex system is needed to ensure stability in a rapidly changing environment $[3,4]$.

The power-grid frequency is the central observable for the control of AC electric power grids, as it directly reflects the balance of the grid: A surplus of feed-in power increases the frequency and a shortage reduces the frequency [5]. Observing the frequency of the power grid can thus provide deep insights into the dynamical stability of the grid as well as the operation of the control system and the economic dispatch of generators. In today's system strict operational boundaries are

\footnotetext{
*anvari@pik-potsdam.de

†1.rydin.gorjao@fz-juelich.de

¥marc.timme@tu-dresden.de

§.witthaut@fz-juelich.de

"b.schaefer@qmul.ac.uk
}

Published by the American Physical Society under the terms of the Creative Commons Attribution 4.0 International license. Further distribution of this work must maintain attribution to the author(s) and the published article's title, journal citation, and DOI. imposed on the frequency and the rate of change of frequency [6]. For example, in the Central European power grid (CE), the stable operational boundary for frequency variations is set at $\pm 200 \mathrm{~Hz}$. Moreover, if the frequency deviates more than $\Delta f= \pm 20 \mathrm{~Hz}$, the existing control systems, i.e., primary and secondary control, are activated to compensate the imbalance in the power grid and to return the frequency to the nominal one [7].

These control mechanisms and operational boundaries are especially interesting when designing new grids involving concepts such as smart grids [8], prosumers [9], or microgrids [10], and their interaction with the grid frequency. Furthermore, due to the increased usage of renewable energies, synchronous machines are replaced by power electronics, such as inverters, posing additional challenges on ensuring frequency stability [11]. Inverter-based generators do not have any innate inertia, leading to the frequency of the power grid becoming more volatile, unless additional stabilizers are included in the system [12].

A more sophisticated analysis of the power-grid frequency dynamics is paramount, as all power generators and consumers have to ensure the stability of the grid in the presence of many effects simultaneously impinging on it. In such analyses it is both relevant to study existing power grids [13] as well as to evaluate any forecasts and models of the frequency dynamics expected in future grids [14].

Despite the strict operational boundaries for frequency variations, numerous different sources of disturbances 
introduce measurable variations of the frequency over time. Important sources introducing fluctuations to the grid frequency include consumers, renewable energies, and the dispatch of power plants via the energy market. Recent research shows that today's demand fluctuations contribute substantially to uncertainties in the power balance [15-17]. Moreover, intermittent renewable energies influence the frequency first due to their stochastic and often non-Gaussian power feed-in $[18,19]$, and second due to the decreasing the inertia in the power grid, as mentioned above. Hence, to operate energy systems with a high share of renewable energies, a solid understanding of the impact of fluctuating feed-in on the grid's frequency is necessary. Previous studies described the stochastic behavior of the grid frequency using stochastic optimization [20], a simulated robustness analysis [21], FokkerPlanck approaches [22,23], or tracing the impact of wind feedin on the grid frequency $[24,25]$, and the integration of storage systems to improve the frequency quality in the presence of wind power [26]. However, the mathematical properties of the underlying stochastic process have not been studied comprehensively.

In addition to the aforementioned stochastic disturbances, trading affects the grid frequency by scheduled deterministic periodic events, e.g., dispatch actions on the energy market cause brief jumps of the frequency $[13,23,27]$. While deterministic disturbances have been observed for various grids $[13,28]$, no comprehensive model exists to describe the market interaction with the grid frequency quantitatively. We thus aim for a dynamical model of the power-grid frequency including the role of trading and regulator action in the power grid. Such a model may help especially to plan future grids with a high share of renewable energies. Volatile renewable energies, such as wind and solar power, are unpredictable and thus cannot be used to balance the grid frequency following trading actions. Instead, it is fundamental to understand the interplay between the stochastic dynamics of unpredictable fluctuations and the deterministic characteristics of the energy market.

Here we first review essential statistical properties and the temporal evolution of the frequency of real-world power grids. With a special focus on the deterministic fluctuations at trading and dispatch times. Our approach provides a method to obtain bountiful information on the power-grid frequency that can be obtained from simple measurements. Next we introduce our stochastic model to regenerate the frequency dynamics and explain how we estimate its parameters solely from the power-grid trajectory. Finally, we demonstrate how our model reproduces key aspects of the stochastic and deterministic behavior of real trajectories.

\section{POWER-GRID FREQUENCY OVERVIEW}

The power-grid frequency displays several characteristic features, such as non-Gaussian distributions, an exponential decay of the autocorrelation, and regular impacts by trading [23]. We extend earlier studies by uncovering other stochastic properties of power-grid frequency, namely addressing the questions of Markovianity, linearity, and stationarity of the data. Specifically, we investigate the recorded frequency from Great Britain (GB) [29], and from two different regions in central Europe (CE). The two data samples of CE have

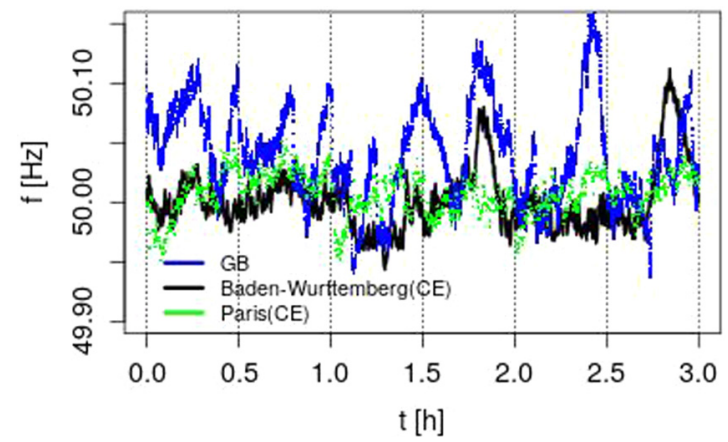

FIG. 1. The power-grid frequency fluctuates over time, with differences between distinct regions. Displayed are $3 \mathrm{~h}$ of frequency trajectories on March 1st for Paris, Baden-Württemberg (both CE), and GB. The data sets belong to 2015, 2016, and 2017, respectively, for Paris, GB, and Baden-Württemberg. Note that the BadenWürttemberg and Paris data are from different years, while still displaying similar statistics.

been recorded in Paris (France) [30] and Baden-Württemberg (southwest of Germany) [31]. The time resolution of data sets are 1, 10, and $1 \mathrm{~s}$, respectively for GB, Paris, and BadenWürttemberg. We analyze data spanning over one year: 2015 for France, 2016 for GB, and 2017 for Baden-Württemberg. The final section addresses the modeling following the data from Baden-Württemberg. A direct observation of the frequency of the three samples (Great Britain, Paris, BadenWürttemberg) during three arbitrarily chosen hours in March reveals substantial differences in the fluctuation patterns, see Fig. 1. The range of variations in GB is larger than in the other two frequency data sets. The reason being, the primary control in GB is only activated for frequency deviations of at least $\pm 200 \mathrm{~Hz}$, while the other frequency sets belong to the CE grid, where control is activated at $\pm 20 \mathrm{~Hz}$. Consequently the CE data set has smaller overall fluctuations and a lower standard deviation.

In contrast to many random processes, the values of the power-grid frequencies do not strictly follow Gaussian (normal) distributions [32,33]. Instead, the distributions display heavy tails, where large deviations occur much more frequently than anticipated from a normal distribution. In Fig. 2 the frequency and increment frequency distributions of GB and Baden-Württemberg are shown. As both Paris and BadenWürttemberg belong to the CE power grid, they have similar (but not identical) statistical properties. Therefore, for the rest of this section, we focus our analysis on the frequency measurements from Baden-Württemberg as an example, and where we aim to refer to general statistic features, we refer to the $\mathrm{CE}$ grid. Comparing the frequency probability distribution function (PDF) with the best-fitting normal distribution, highlights the non-Gaussian properties of the frequency PDF of CE, which has a kurtosis 4.23, Fig. 2(c). The kurtotsis, the normalized fourth moment, measures the heavy-tailedness of a distribution, see, e.g., [34]. Any value of the kurtosis larger than the that of a normal distribution $\left(\kappa_{\text {normal }}=3\right)$ indicates heavy tails [35]. The frequency distribution for GB breaks the symmetry expected from a normal distribution and exhibits a skewness of 0.191, see Fig. 2(a). The skewness, the normalized third moment $\beta$, measures how skewed, i.e., 

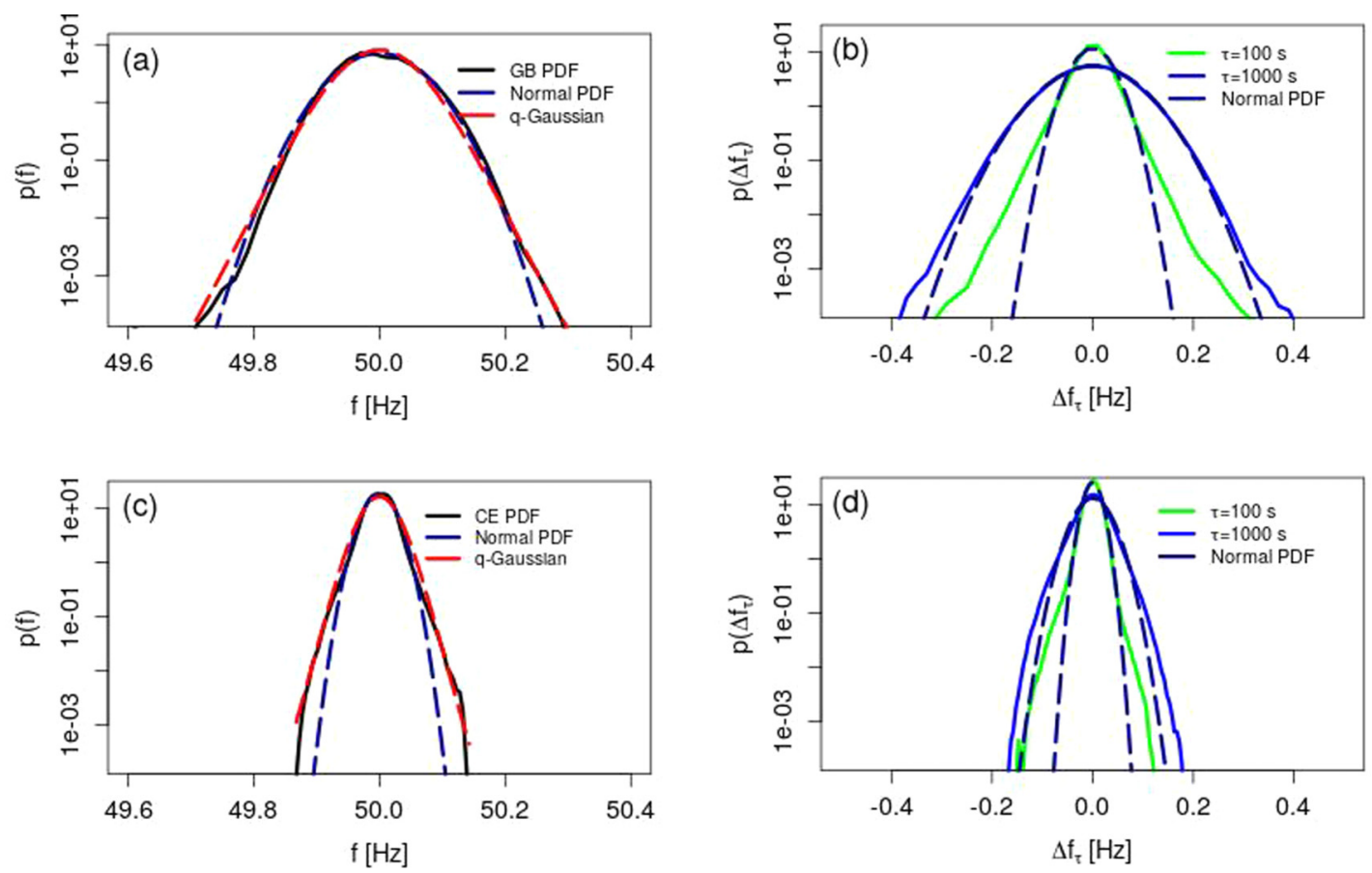

FIG. 2. Both the PDFs of the frequency and of the frequency increments display non-Gaussian features. We compare the PDF of the frequency with the most-likely Gaussian fit (blue curve) and q-Gaussian (red curve), for (a) the GB grid and (c) the CE grid evaluating the Baden-Württemberg time series. We observe an asymmetry (nonzero skewness $\beta$ ) in the GB data with the deformation parameter $q=0.95$ and pronounced heavy tails (high kurtosis $\kappa$ ) in the CE data with $q=1.1$. Increment statistics in (b) GB and (d) CE grid were carried out for different time lags. Short-time lag $(\tau=100 \mathrm{~s})$ displays more pronounced deviations from Gaussianity (dashed lines) than larger time lags.

asymmetric, a distribution is. For a normal distribution, the skewness is zero. Furthermore, based on the shape of the PDFs, large deviations of the power-grid frequency towards very low frequencies occur more often in the GB grid, while deviations towards higher frequencies are more common in the CE grid. We note that both skewness and kurtosis statistics depend on the sample size, but the observed non-Gaussian features are genuine since we do use large data sets with high sample frequency. Instead of normal distributions, the observed statistics is possibly better described by Lévy-stable or q-Gaussian distributions [23].

The frequency increment statistics also display nonGaussian features. We estimate the probability to observe large fluctuations on short timescales by computing frequency increments, i.e., $\Delta f_{\tau}=f(t+\tau)-f(t)$, see Figs. 2(b) and 2(d), for $\tau=100 \mathrm{~s}$ and $\tau=1000 \mathrm{~s}$, respectively. Next, we compare the observed increment probabilities with the best Gaussian fit: Frequency variations of the order of $210 \mathrm{mHz}$ within $100 \mathrm{mHz}$ occur in the GB frequency data set $10^{5}$ times more often than expected for Gaussian processes. For the Baden-Württemberg data, frequency variations $\sim 60 \mathrm{mHz}$ occur 100 times more often compared to a Gaussian distribution. The increment frequency statistics indicates that the frequency on the short timescale is particularly subject to large fluctuations. Potentially new control systems or market mechanisms are necessary to compensate the power imbalance in the power grid on short timescales. In contrast, the shape of the frequency and frequency increment PDF become similar for larger time lags, such as $\tau=1000$, and the deviation from Gaussianity is not as extreme as for the short timescale, see Figs. 2(b) and 2(d).
To obtain more information from the frequency trajectory, we investigate the autocorrelation and its decay for the frequency data sets. The autocorrelation measures the correlation of a signal with itself at a later time. High correlation values indicate that a large signal is typically followed by still a large signal and vice versa. The power-grid frequency autocorrelation decays approximately exponentially as a function of the time lag $\Delta t$ for short-time lags, see [23] and Fig. 3. Several prototypical stochastic processes, such as the Ornstein-Uhlenbeck process, display a similar decay, following precisely an exponential function [36]

$$
\begin{aligned}
& c(\Delta t)=\langle f(t) f(t+\tau)\rangle, \\
& c^{\mathrm{OU}}(\Delta t)=\exp (-\alpha \Delta t),
\end{aligned}
$$

with a damping constant $\alpha$. While initially the system is highly correlated with its own history, this damping will cause a decorrelation. Naturally, distinct power grids will have their specific characteristic damping constant. A least squares fit of an exponential decay (2) to the data yields $\alpha^{-1}$ which is $\sim 385 \mathrm{~s}$ for the GB grid and $\sim 312 \mathrm{~s}$ for the CE grid respectively, see Fig. 3(a).

Another feature of the autocorrelation are the regular peaks every $15 \mathrm{~min}$, which are highlighted with black arrows in Fig. 3. These peaks are caused by a mismatch of power supply and demand [13,27,32]. In most electricity grids the operation of dispatchable power plants is scheduled in $1 \mathrm{~h}$ blocks, where additional (shorter) 30 and 15 min intervals might exist. Hence the generation curve is steplike, while the demand varies continuously. From step to step, the power 



FIG. 3. Regular peaks in the autocorrelation demonstrate a mismatch between power supply and demand. (a) The autocorrelation $c(\Delta t)$ of GB and $\mathrm{CE}$ for a $1 \mathrm{~h}$ lag period. The black arrows indicate the times of trading/dispatch actions after 15 and $30 \mathrm{~min}$, which cause the peaks in the autocorrelation. The dotted red line reports the exponential decay of the autocorrelation in the first $10 \mathrm{~min}$. The inverse damping constants $\alpha^{-1}$ are estimated to be $\sim 385$ and $\sim 312 \mathrm{~s}$ for the GB and CE power grids, respectively. (b) The autocorrelation function $c(\Delta t)$ of the GB (black) and CE (blue) data sets for a $24 \mathrm{~h}$ lag period. Regardless of regions, the initial exponential decay is followed by regular autocorrelation peaks. The black arrows highlight peaks of the autocorrelation after $1 \mathrm{~h}$ and and also after $24 \mathrm{~h}$, related to the periodicity of the frequency trajectory.

balance rapidly switches from positive to negative or vice versa, leading to large deviations of the grid frequency, which become visible in the autocorrelation function, see also [14]. In addition, daily routine, scheduled events, etc., contribute to an increased correlation every hour and $24 \mathrm{~h}$, see black arrows in Fig. 3(b). Again, based on the specific regulations of different synchronous regions and their transmission system operators, the nature of the autocorrelation differs from region to region. For instance, the height of peaks in the GB autocorrelation in Fig. 3(a) is visibly smaller than CE, which we attribute to a smaller trading and regulatory volume and overall larger stochastic fluctuation in GB. Consequently, the deterministic aspect of the frequency dynamics is diluted in GB.

Finally, to clearly demonstrate the impact of the energy trading market and related regulator actions on the frequency, we show the daily average frequency of both GB and CE in Fig. 4. The daily average frequency for every second is obtained by averaging over all days of the year. The impact of the trading and regulation becomes clear, as we observe sharp frequency jumps upwards or downwards every hour in both GB and CE. The direction of the jump and thereby the
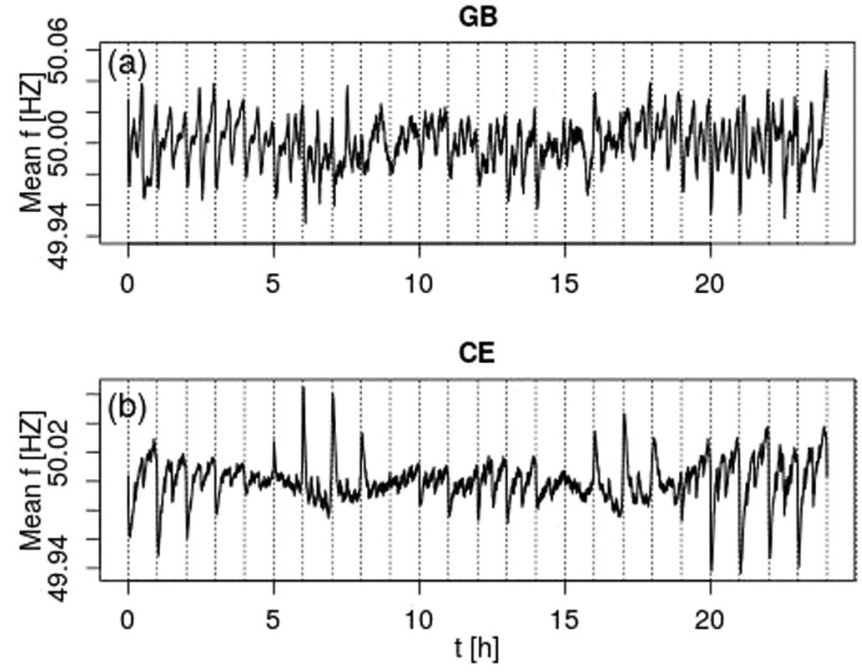

FIG. 4. Regular market activities induce periodic frequency jumps. Displayed is the frequency trajectory for (a) the GB grid and (b) CE grid, averaged over all 366 days in 2016. We notice clear frequency jumps every hour, consistent with the previous observation of peaks in the autocorrelation function.

question whether the grid is displaying a shortage or a surplus of power is not random but also follows a deterministic pattern. The market design is different for various synchronous grids or different countries within the same grid. For example, both the CE and the GB data display a periodicity of frequency jumps but the frequency dynamics within the CE grid appears more predictable. Frequency drops occur in the CE grid in each hour between 20:00 and 00:00, while the frequency clearly increases between 06:00 to 08:00 and 16:00 to $18: 00$. This pattern is linked to the slope of the demand curve. The steplike generation curve anticipates an increase or decrease of the demand [13]. In case of rising demand, such as during the morning, an increasing amount of power is dispatched for each trading interval, see Fig. 5(b). Every $15 \mathrm{~min}$ the generation is increased to anticipate the demand by the consumers. These discrete changes in the supplied power form the basis for the power mismatch in the synthetic frequency model discussed below.

\section{STOCHASTIC PROPERTIES}

Before we introduce a stochastic model for the power frequency dynamics, we perform some complementary tests to further characterize the underlying stochastic dynamics. Is the observed stochastic process stationary or nonstationary? Do we observe time symmetry, i.e., is the underlying process linear or nonlinear? Does the process depend on its past or only on the current state, i.e., is the process Markovian?

Stationary process. To test the reproducibility of the measured frequency, we first investigate the stationarity for the data. In the general definition, a probabilistic process is stationary if the probability of measured variables, in our case the probability of the frequency, does not depend on the time [38]. One of the standard methods to test the stationarity of a data set is analyzing its spectrum. The sharp peaks in Fig. 6 emphasise the existence of the periodicity on different 



FIG. 5. Discrete power dispatch leads to jumps of the scheduled power supply. (a) We display the real dispatch trajectory of the electricity supply in Germany in one day in 2017 [37]. (b) The scheduled power jumps every 15 min, as highlighted by the zoom on the two hours highlighted in red in (a). Overall, the scheduled power supply approximates the changing demand throughout the day. Its discrete nature leads to jumps of the supply that has to be compensated by control mechanisms.

timescales in the considered data. According to the spectrum, there are visible periods every $1 / 4,1 / 2,1,12$, and $24 \mathrm{~h}$ in the grid frequency. This shows the nonstationary of the data on these timescales. However beyond 24 h, i.e., on longer timescales, the spectrum is decreasing and consequently the data becomes stationary.

There are other natural cycles influencing a power-grid system, such as the weekend-weekday pattern, as well as seasonal and yearly cycles. However, these cycles do not

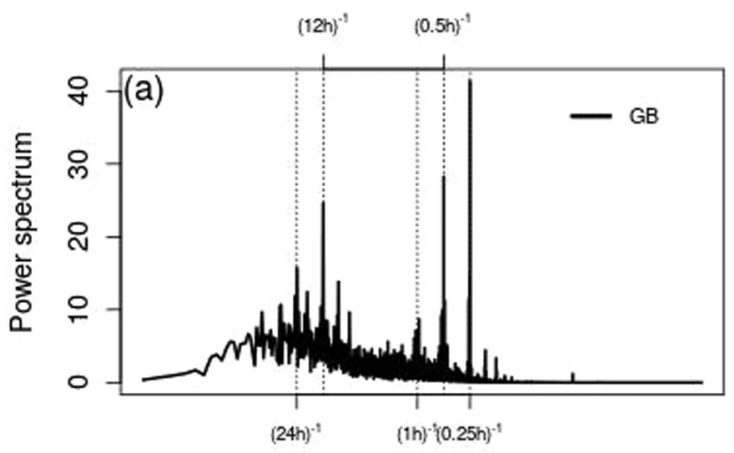

frequency

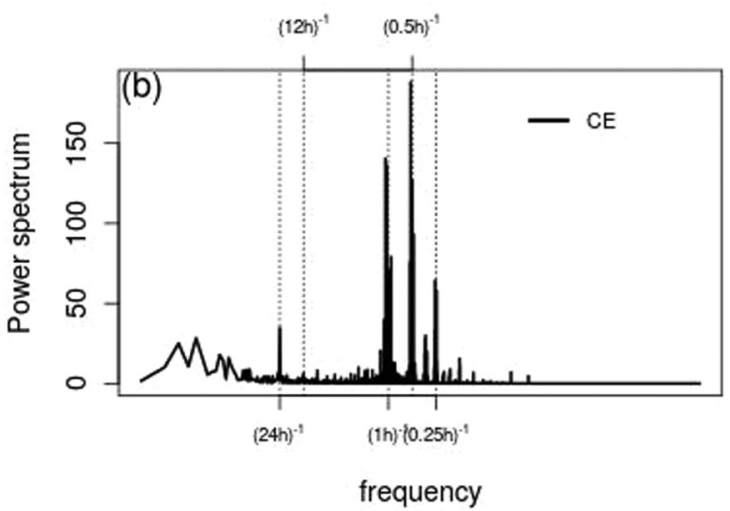

FIG. 6. Market activities and long timescales introduce nonstationarity. We plot the power spectrum of (a) GB and of (b) the CE data. The spectra exhibit well-determined peaks before they decay on a large timescale. The dotted vertical lines show $1 / 4,1 / 2,1,12$, and $24 \mathrm{~h}$ cycles (from right to left). seem to leave a significant imprint in the spectrum of the power-grid frequency. Our stochastic model will focus on the intermediate timescale and hence include the characteristic daily dispatch and demand pattern, while neglecting longerterm processes.

Linear process. Next, we investigate if there is any nonlinearity in the recorded power-grid frequency. For this purpose, consider the three-point autocorrelation of the frequency data as a measure of the time asymmetry in the data. If a time series is asymmetric in time, it is also nonlinear [38]. The following relations have been suggested to calculate the three-point autocorrelation for a data set [38]:

$$
\begin{gathered}
L T 1=\left\langle f(t)^{2} f(t+\tau)\right\rangle-\left\langle f(t) f(t+\tau)^{2}\right\rangle, \\
L T 2=\left\langle[f(t)-f(t+\tau)]^{3}\right\rangle /\left\langle[f(t)-f(t+\tau)]^{2}\right\rangle,
\end{gathered}
$$

where $L T$ stands for linear test. A linear, and therefore timesymmetric, trajectory has both $L T 1$ and $L T 2$ sufficiently close to zero. Checking the validity of our results for a realistic process, we compare the original data to a surrogate time series, that provides a reference point of $L T 1$ and $L T 2$ for a linear process. To generate the surrogate time series, we first take the Fourier transform (FT) of the original data and then randomize the phases. Finally, we employ an inverse FT to obtain the surrogate data. With the described procedure we suppress any nonlinearity in the process, and therefore the surrogate data includes only the linear characteristics of the considered data [39]. The original data is linear if the $L T$ result of the original data lies within the value range of the $L T$ results of the ensemble of surrogate data. Here, instead of displaying the full ensemble of surrogate data in Fig. 7, we have shown just an example for a surrogate data to avoid to obscure the figure. Comparing the $L T 1$ results of the surrogate data sets with the $L T 1$ of the original data sets displays that the qualitative behavior of both are equivalent, entailing that the processes approximately follow linear characteristics, for both the GB and the CE data sets, as seen in Fig. 7(a). Looking more closely at the $L T 1$ for the CE surrogate data, which only includes the linear characteristics and fluctuations, we note that its deviation from zero are larger than $L T 1$ for the original CE data. Investigating the value of $L T 2$ for GB also confirms the linear characteristic of the data set. As the $L T 1$ and $L T 2$ 

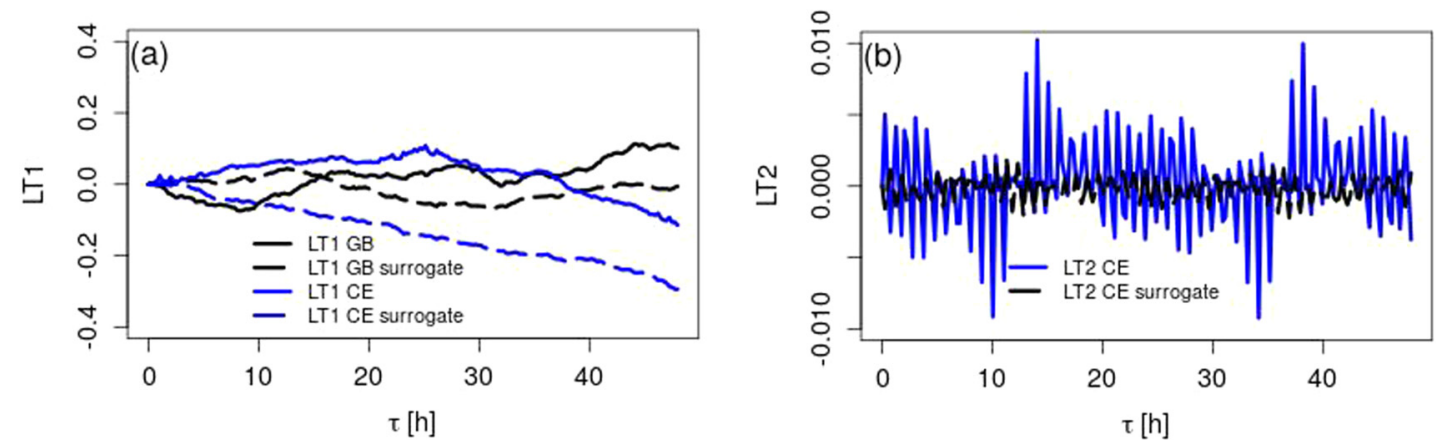

FIG. 7. The frequency trajectories display small nonlinear effects. (a) The LT 1 results for the GB and CE frequency measurements. The dashed lines show the $L T 1$ results for the surrogate data sets. The surrogate results act as a reference case of a linear model. Comparing the results of the original data with surrogate ones, we conclude both GB and CE are approximately linear. (b) $L T 2$ results for the CE data. Surrogate (dashed black) and original data (solid blue) do differ more than when using $L T 1$. This difference and the periodicity in $L T 2$ are the signature of small nonlinear effects.

results for GB are the same, we only show the $L T 1$ results. However, for the CE data set, LT2 indicates that the data might not be strictly linear but displays small nonlinearities, as seen in Fig. 7(b). As shown in Fig. 3, the effect of the market activity in $\mathrm{CE}$ is more regular and more severe than in GB, therefore we suspect that the nonlinearity in CE data is caused by the regular jumps in the frequency trajectory. When devising our model, we will therefore approximate the weakly nonlinear process as linear.

Chapman-Kolmogorov test. A fundamental property of stochastic processes is whether future states only depend on the current state or whether they have memory. In other words, whether the process is Markovian or not. A well-known approach to evaluate whether a process is Markovian is the Chapman-Kolmogorov test [36]. According to the ChapmanKolmogorov test, the conditional PDFs of Markovian processes obey the following equation:

$$
p\left(f_{3}, t_{3} \mid f_{1}, t_{1}\right)=\int p\left(f_{3}, t_{3} \mid f_{2}, t_{2}\right) p\left(f_{2}, t_{2} \mid f_{1}, t_{1}\right) d f_{2},
$$

where $t_{3}>t_{2}>t_{1}$. To test the Markovianity for the data, instead of employing directly Eq. (5), one considers its 2D and 3D conditional PDF. As shown in Fig. 8, $p\left(f_{3}, t_{3} \mid f_{1}, t_{1}\right)$ and $p\left(f_{3}, t_{3} \mid f_{2}, t_{2} ; f_{1}, t_{1}\right)$ match approximately, implying the power-grid frequency is mostly Markovian. Any stochastic model for the power frequency should therefore be Markovian as well.

\section{STOCHASTIC MODEL}

We now introduce a synthetic model for the power-grid frequency as a stochastic, mostly linear, and Markovian process. The stochastic model presented here aims at reproducing essential features of a power grid, as well as its statistical characteristics, and consists of three independent systems: First, the intrinsic deterministic dynamics of the power grid, including primary and secondary control. Second, it embodies as well a stochastic signal or noise, as evidenced by the aforementioned frequency trajectories [27]. Third, we model the sudden power imbalance arising after the dispatch actions by implementing an appropriate deterministic function: We make use of historic dispatch data and apply it using a a step function of the power. Other functions, such as artificial steps or sawtoothlike functions are also possible.

Instead of the actual frequency, we use the bulk angular velocity relative to the reference frequency of $50 \mathrm{~Hz}, \omega=$ $2 \pi(f-50 \mathrm{~Hz})$ to express our model. Contrary to network analysis on power grids [40,41], we have only access to
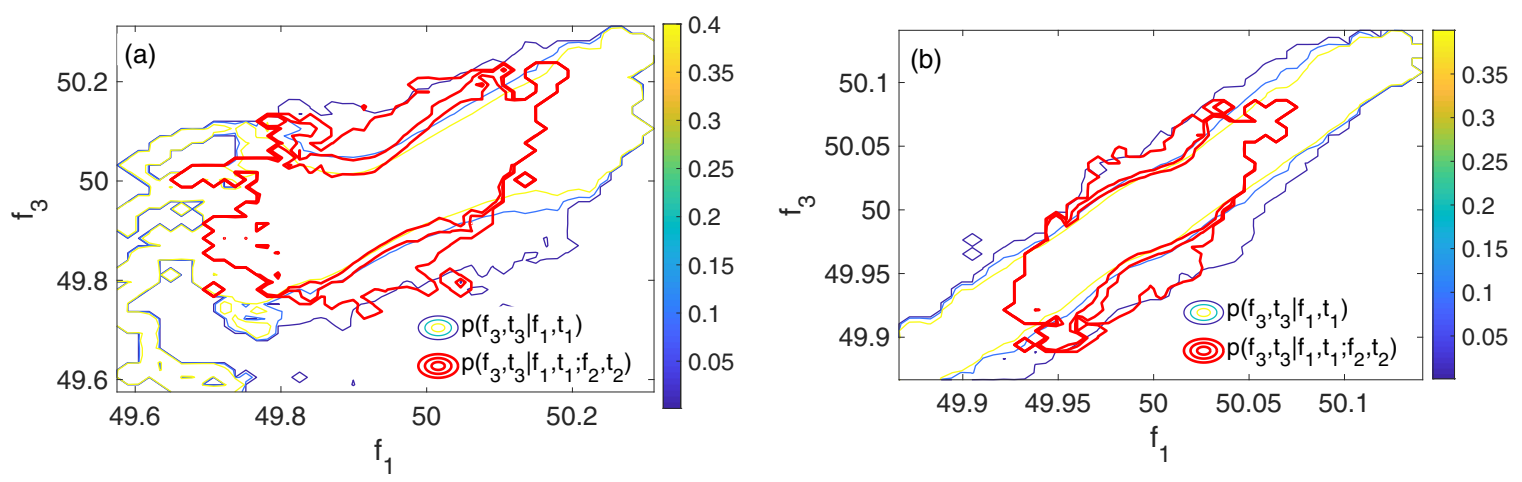

FIG. 8. The Markovian nature of the real data is confirmed by a Chapman-Kolmogorov test for (a) the GB grid and (b) the CE grid using the Baden-Württemberg data set. The proximity of the contour lines of $p\left(f_{3}, t_{3} \mid f_{1}, t_{1}, f_{2}, t_{2}\right)$ (red contour) and $p\left(f_{3}, t_{3} \mid f_{1}, t_{1}\right)$ (colored contour) show the validity of Chapman-Kolmogorov test for the frequency data sets. The time $t_{1}$ is chosen to contain ten data points to show the contours clearly. Next, the times $t_{2}$ and $t_{3}$ are multiples of $t_{1}$, chosen as $2 t_{1}$ and $3 t_{1}$, respectively. 

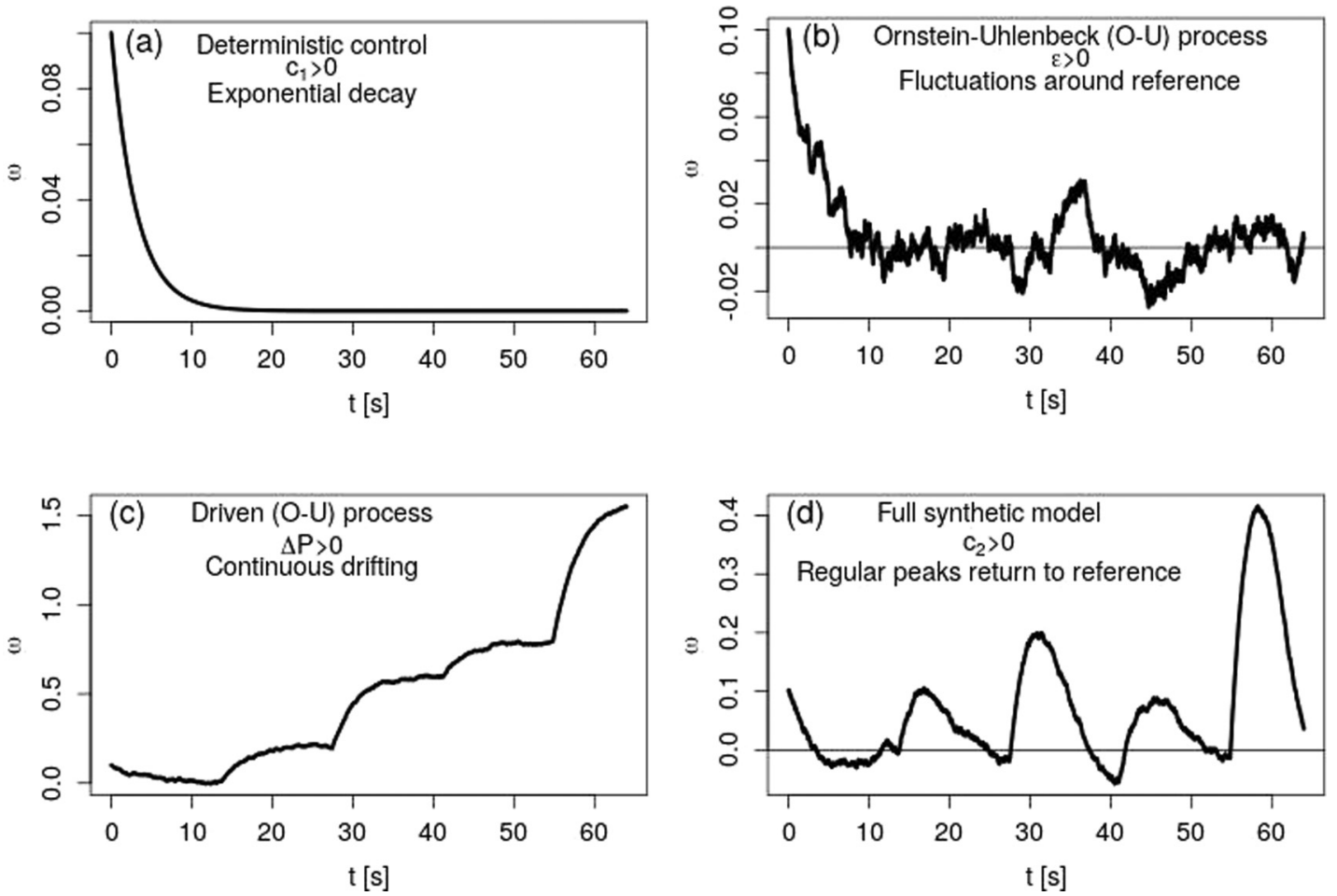

FIG. 9. All terms of the synthetic model (6) are necessary to reproduce the frequency trajectory. We plot the angular velocity $\omega$ as a function of time when using the synthetic frequency model (6) but setting individual parameters to 0 . Parameters are chosen for pure illustrative purpose and we set $\omega(0)=0.1$ as an initial condition. (a) Including only primary control leads to a pure exponential decay of the angular velocity. (b) Adding nonzero noise $\epsilon$, we recover an Ornstein-Uhlenbeck process. (c) Including a step function for the power imbalance $\Delta P$ leads to a continuously drifting Ornstein-Uhlenbeck process. (d) Finally, including secondary control guarantees that the angular velocity returns back to the reference. Parameters are $\epsilon=0.001 / s^{2}, c_{1}=0.005 / s, c_{2}=0.00003 / s^{2}, \Delta P=0.004 / s^{2}$ at every hour and half or a quarter of it every 30 or 15 and $45 \mathrm{~min}$, respectively.

frequency measurements on the global scale and therefore average over all nodes to obtain the averaged (bulk) frequency and angular velocity [42] $\omega=\frac{1}{M} \sum_{i=1}^{N} M_{i} \omega_{i}$, where $M=\sum_{i=1}^{N} M_{i}$ is the total inertia of all nodes and $N$ is the number of nodes in the power grid. Typically, the frequency at each node is very close to the bulk frequency throughout the grid, with fluctuations indicating the gross power balance. Notable exceptions are high-frequency disturbances, which are typically localized $[43,44]$, or interarea oscillations, where energy is oscillating from one part of the grid to another one. The synthetic model of the frequency dynamics is discussed in detail in [14]. It is given as a linear stochastic differential equation:

$$
\frac{d \omega}{d t}=-c_{1} \omega-c_{2} \theta+\Delta P_{\mathrm{ext}}+\epsilon \xi,
$$

with bulk angle $\theta$ and its derivative $d \theta / d t=\omega$. Furthermore, $\Delta P_{\text {ext }}$ is the exogenous influence on the power balance, i.e., the trading or dispatch impact of the power imbalance, $\epsilon$ and $\xi$ are the noise amplitude and Gaussian white noise function, respectively. Finally, $c_{1}$ is the magnitude of the fast-acting primary control, while $c_{2}$ is the magnitude of the secondary control which acts slower and lasts longer than primary control. We illustrate the contribution of the different terms of the synthetic model (6) in Fig. 9.

The full model is displayed in Fig. 9(d): In case of an abundance of generation, i.e., a sudden positive $\Delta P_{\text {ext }}$, the frequency increases above the reference $(50 \mathrm{~Hz})$. The primary control $c_{1}$ mitigates the sudden rise of the frequency and quickly stabilizes the frequency, but not at the nominal value of $50 \mathrm{~Hz}$. Subsequently, the secondary control slowly restores the frequency back to its reference of $50 \mathrm{~Hz}$. According to the time schedule of control systems, we assume that the primary control acts faster than secondary control, and consequently $c_{1} \gg c_{2}[45,46]$.

Furthermore, the nature of the dispatch structure $\Delta P_{\text {ext }}$ must be specified. The generation of each power plant (the dispatch) is rapidly adapted by the operators, e.g., based on trading at the European Energy Exchange. As discussed in detail at the end of Sec. II, the operation of dispatchable power plants is scheduled at fixed intervals. As we have shown in Fig. 5 the power generation can increase or decrease every 15 min, which we model approximately as a step function, with potentially different step sizes at the $1 \mathrm{~h}, 30 \mathrm{~min}$, or 15 min intervals. On the other hand, data of power generation in different regions or countries are generally available, and can be implemented directly in the model. In the model presented here, we extracted the power generation in Germany for the equivalent month of December 2017, and used this as the power balance $\Delta P_{\text {ext }}[37]$.

Before we compare results of the synthetic model with the real data, we need to determine suitable parameters. Details are given in [14] on how to estimate the parameters from a given frequency trajectory. In short, the noise amplitude 
TABLE I. The parameters for the synthetic model for CE, December 2017.

\begin{tabular}{lll}
\hline \hline$\epsilon\left(s^{-2}\right)$ & $c_{1}\left(s^{-1}\right)$ & $c_{2}\left(s^{-2}\right)$ \\
\hline 0.00107 & 0.00915 & 0.00003 \\
\hline
\end{tabular}

$\epsilon$ is estimated based on the stochastic fluctuations around the observed frequency trajectory, while the power imbalance $\Delta P_{\text {ext }}$ is directly read from the rise or sag of the frequency at the scheduled time points of dispatch, which are proportional to the missing or exceeding amount of power. (Notice that in our case we include the real power generation from Germany for December 2017, thus circumvent extracting the power generation $\Delta P_{\text {ext }}$ from the data.) Primary control $c_{1}$ is recovered by studying the process' affinity to revert its trajectory to the dispatched power and secondary control $c_{2}$ is estimated from the frequency recovery rate to the nominal value after a scheduled action [14].

\section{QUANTITATIVE COMPARISON BETWEEN MODEL AND DATA}

To evaluate the stochastic model described above, we generated one month of synthetic data with a 1-s resolution, mirroring the CE data from December 2017. The parameters for the synthetic model [14] are estimated from the 1-s resolution data series provided by [31] and their values are shown in Table I. The data for the power generation for the month of December 2017, in Germany, can be found in [37].

Now we repeat most of our statistical and stochastic analyses to compare how well the synthetic model reproduces the original data. First, we note that the general shape of the PDF [see Fig. 10(a)] and autocorrelation [see Fig. 10(b)] do agree well between the model (yellow) and the empirical data (black). Both the model and the data display heavy tails, i.e., the aforementioned deviation from Gaussianity. Furthermore, the autocorrelation function of the synthetic model captures the regular peaks, due to the changing dispatch. The decay of the autocorrelation function is approximately described by the current model. Both results emphasise the enormous impact of the energy market activity and dispatch structure on the dynamics and stability of the power system.

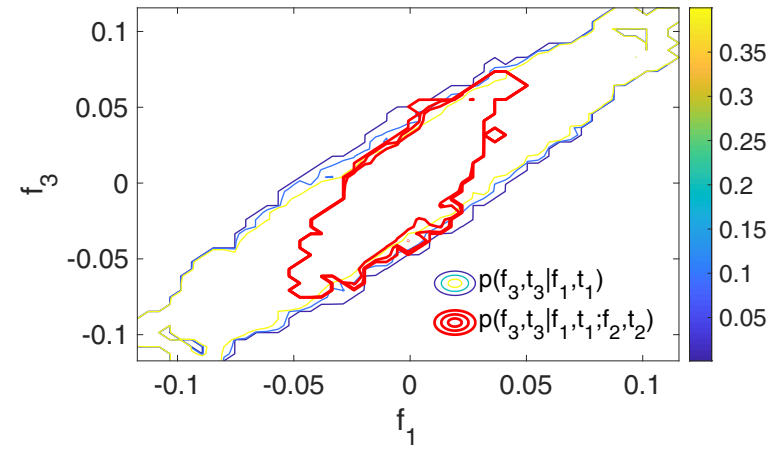

FIG. 11. Chapman-Kolmogorov test confirms the Markovian nature of the synthetic model. The test used 1 month of synthetic CE data generated by (6).

Consistent with our modeling assumptions, we find that the synthetic model is Markovian, based on a ChapmanKolmogorov test, see Fig. 11. Similarly, we do observe that both $L T 1$ and $L T 2$ results show that the synthetic model has compatible characteristics with the real one, i.e., while the $L T 1$ reports a linear process, $L T 2$ results show a small nonlinearity in the synthetic, cf. Fig. 12. As we discussed in Sec. III, this nonlinear behavior is likely linked to the regular trading in the CE power grid.

We again emphasise that our model addresses the dynamics on the intermediate timescale of the frequency, i.e., approximately $30 \mathrm{~s}$ to a few hours. On shorter timescales, our model neglects: (i) dynamical behavior of rotating machines, (ii) nontrivial stochastic noise, (iii) network dynamics, and (iv) momentary reserve vs primary control. Moreover, the switching in trading is not instantaneous as we have assumed in the model. Similarly, our model does not include all effects acting on larger timescales, for example, (i) feed-in of wind and solar power, which determines how much inertia exists in the system and how much the generation side fluctuates, and (ii) dispatch of power plants determined on the spot market, such as the European Energy Exchange (EEX). This is especially relevant for areas where no historic market data are available or forecasts are attempted. In order to capture these effects, we would need a full fledged market model plus meteorological input for the weather data.
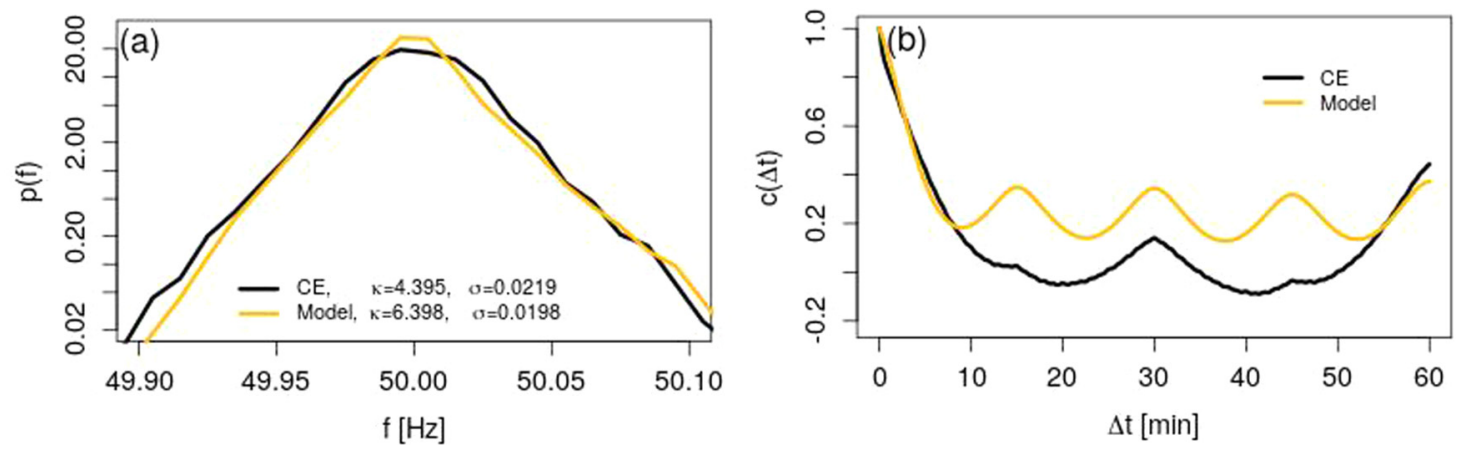

FIG. 10. The synthetic model captures important features of the real data, including trading peaks and heavy tails. (a) The probability distributions of the frequency data from CE in 2017 (black), compared to our synthetic model (yellow). Both display distinct heavy tails with kurtosis $\kappa>3$. (b) The autocorrelation function of the frequency initially decays and then displays regular peaks at the trading intervals. 


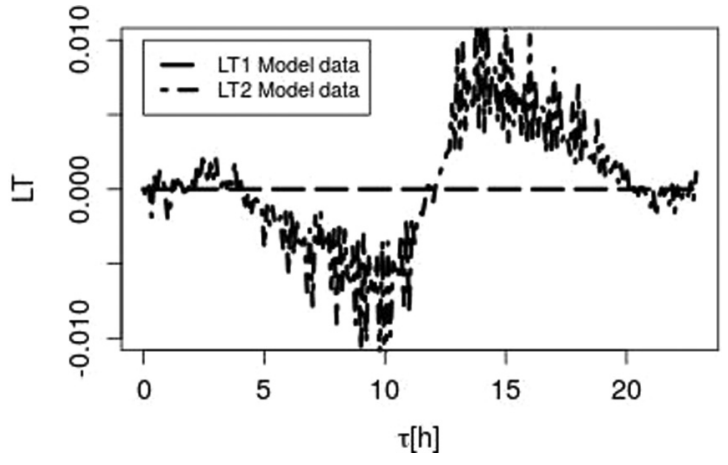

FIG. 12. The synthetic model is approximately linear. We apply the linear tests on the time series generated by the synthetic model: $L T 1$ shows linear characteristics for the CE data set, however $L T 2$ reports a small nonlinearity also found in the real data of the $\mathrm{CE}$ power-grid frequency.

The spectral analysis and the increment statistics of the synthetic data are shown in Fig. 13. Similarly to Fig. 2, in Fig. 13(a) the frequency increment statistics of the generated data also display non-Gaussian features on short timescales as the real data. The spectrum of the synthetic frequency trajectory displays several pronounced peaks, which are mostly
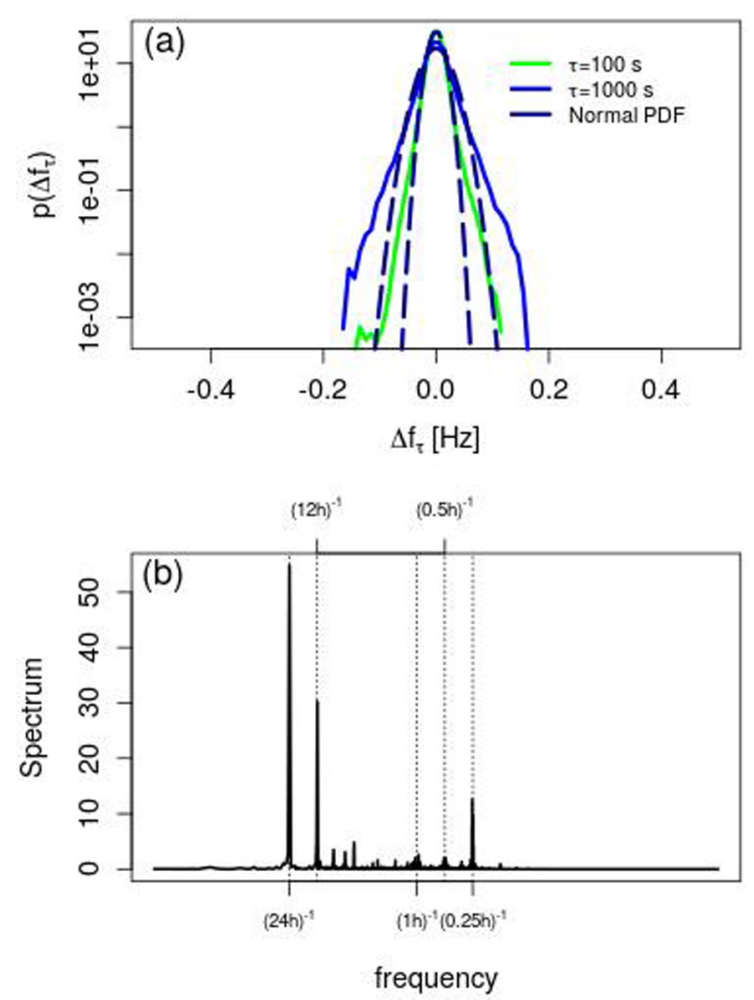

FIG. 13. Increment and spectral analysis of the synthetic model are consistent with the real data. (a) The increment statistics of the synthetic data shows non-Gaussian characteristics similar to the real one. (b) The spectrum of the synthetic frequency trajectory reports large peaks at the trading times, while it decays to zero for longer timescales. The dotted vertical lines show respectively, $1 / 4,1 / 2,1$, 12 , and $24 \mathrm{~h}$ from right to left. consistent with the trading times of the model, i.e., 1/4, 12, and $24 \mathrm{~h}$ (cf. Fig. 13).

\section{DISCUSSION}

In summary, we have presented an analysis of the statistics of power-grid frequency dynamics, with an emphasis on nonstandard behavior. In particular, we have shown the nonGaussian nature of the power-grid frequency fluctuations in the aggregated and increments statistics, which includes heavy tails. Furthermore, we have demonstrated that the power-grid frequency trajectory is adequately described as a Markovian process and that it shows small nonlinear effects. Regulatory and trading events introduce some distinct periodicities in both autocorrelation and spectrum of the data sets. As we have mentioned before, the trading also has an obvious effect on the tails of frequency PDFs, or in other words, it is the source of non-Gaussinaity in the measured data [27]. Finally, based on the observed properties, we have constructed a synthetic model that captures not only the aggregated statistics in terms of the histogram but also qualitatively reproduces the observed autocorrelation decay, correlation peaks due to market activity, increment statistics, and spectral properties of the real data [14]. The model is well suited to understand the energy-market effects on power-grid frequency on intermediate timescales and goes beyond previous studies focusing on a description [13,27] of trading or a stochastic theory [23]. We here focused on a statistical and stochastic analysis of real-world frequency dynamics, with a comparison to the presented model. The analysis of the synthetic model is consistent with our modeling assumptions, in that it is approximately Markovian and displays small nonlinear and periodic market effects. We should emphasise here that the observed heavy tails of the frequency distributions arise mainly due to trading actions, impacting not only the frequency temporally close to the market action but also several minutes later. This is clear since we only applied Gaussian noise to an otherwise linear dynamics. Only the deterministic trading actions can therefore cause the non-Gaussian properties. The spectral and increment properties of the synthetic model also approximate the original real-world data, which confirms again the effect of the trading market on the frequency dynamics. It is worth to reiterate that the presented model is conceptually simple, easy to implement, and includes a minimum set of adjustable parameters. Therefore, we explicitly did not model the machine dynamics, noise on very short timescales or a detailed market and dispatch model. Some alternative model approaches, involving more fitting parameters are explored in [14].

Concluding our analysis of power-grid frequency dynamics and the stochastic model we presented, including a structured comparison, may help to better understand the interplay of the internal dynamics and external disturbances of electricpower systems and to develop improved simulation models. A thorough understanding of this interplay is a prerequisite for the design and optimization of future electricity markets, as well as regulatory and control schemes. For instance, the current market design in the continental European grid regularly causes substantial frequency deviations when the dispatch is adjusted every $15 \mathrm{~min}$ such that primary control 
has to be activated on a regular basis. A smoother change of the dispatch could reduce these frequency deviations and reduce stress onto the primary and secondary control system [13]. Alternatively, frequency regulations could be adapted in a way that the typical frequency deviations due to the changing dispatch are tolerated while exceptional cases are identified and handled by the control system. Our structured analyses (Markov, stationary, and linearity properties) and model may offer a powerful and versatile framework to study these questions, in particular because the model, while still simple, simultaneously captures essential features of the interplay of internal dynamics, control, and market activity. The presented analysis and modeling framework can thus contribute to the design of future power system, reducing the necessity for control actions and saving costs.

The model can further be used to assess the frequency stability of future power-grid structures, including in particular microgrids [8] or low-inertia grids [12]. Traditional dynamical stability analyses focus on local and global stability of fixed points and the impact of large isolated disturbances such as the sudden shutdown of the power plant. In comparison, the impact of ongoing stochastic disturbances on grid stability has received less attention. As evidenced in this study, the regulatory system and market design may have played an important role for these external stochastic effects.
We kept the model as simple as possible to reproduce key features of the frequency time series such as the histogram and the autocorrelation. Future research could naturally extend the model to better match the spectrum or long-time autocorrelation. Furthermore, one could investigate particular intervals of the power grid trajectory, e.g., high- vs low-demand intervals, such as weekdays vs weekends. Additional stochastic investigations could further quantify the agreement between real data and the synthetic model, e.g., by investigating higher-order $\mathrm{N}$ point statistics, going beyond our current two-point statistics (increments).

\section{ACKNOWLEDGMENTS}

We gratefully acknowledge support from the Federal Ministry of Education and Research (BMBF Grants No. 03SF0472F and No. 03EK3055F), the Helmholtz Association (via the joint initiative "Energy System 2050-A Contribution of the Research Field Energy" and Grant No. VH-NG-1025), and the German Science Foundation (DFG) by a grant toward the Cluster of Excellence "Center for Advancing Electronics Dresden" (cfaed). This project has received funding from the European Union's Horizon 2020 research and innovation programme under the Marie Skłodowska-Curie Grant Agreement No. 840825 .
[1] B. H. Obama, Presidential policy directive 21: Critical infrastructure security and resilience, Washington, DC (2013).

[2] J. Markard, The next phase of the energy transition and its implications for research and policy, Nat. Energy 3, 628 (2018).

[3] M. Timme, L. Kocarev, and D. Witthaut, Focus on networks, energy and the economy, New J. Phys. 17, 110201 (2015).

[4] C. D. Brummitt, P. D. H. Hines, I. Dobson, C. Moore, and R. M. D'Souza, Transdisciplinary electric power grid science, Proc. Natl. Acad. Sci. USA 110, 12159 (2013).

[5] P. Kundur, Power System Stability and Control (McGraw-Hill, New York, 1994), Vol. 7.

[6] ENTSO-E, Network code on requirements for grid connection applicable to all generators (rfg) (2013), https://www.entsoe.eu /major-projects/network-code-development/requirements-forgenerators/.

[7] J. Machowski, J. Bialek, and J. Bumby, Power System Dynamics: Stability and Control (John Wiley and Sons, Chichester, 2011).

[8] X. Fang, S. Misra, G. Xue, and D. Yang, Smart Grids-The new and improved power grid: A survey, Commun. Surveys Tutorials IEEE 14, 944 (2012).

[9] P. Kotler, The Prosumer movement: A new challenge for marketers, Adv. Consumer Res. 13, 510 (1986).

[10] R. H. Lasseter and P. Paigi, Microgrid: A conceptual solution, in 2004 IEEE 35th Annual Power Electronics Specialists Conference (IEEE Cat. No. 04CH37551), Aachen, Germany (IEEE, 2004), Vol. 6, pp. 4285-4290.

[11] P. C. Böttcher, A. Otto, S. Kettemann, and C. Agert, Time delay effects in the control of synchronous electricity grids, Chaos $\mathbf{3 0}$, 013122 (2020).

[12] F. Milano, F. Dörfler, G. Hug, D. J. Hill, and G. Verbic, Foundations and challenges of low-inertia systems, in 2018
Power Systems Computation Conference (PSCC) (IEEE, New York, 2018).

[13] T. Weißbach and E. Welfonder, High frequency deviations within the European power system-Origins and proposals for improvement, in Proc. Power Syst. Conf. Expo., Seattle, WA, USA (IEEE, 2009), pp. 1-6.

[14] L. R. Gorjão et al., Data-driven model of the power-grid frequency dynamics, in IEEE Access 8, 43082 (2020), doi: 10.1109/ACCESS.2020.2967834.

[15] A. J. Wood, B. F. Wollenberg, and G. B. Sheblé, Power Generation, Operation and Control (John Wiley and Sons, New York, 2013).

[16] A. Einfalt et al., Energie der zukunft publizierbarer endbericht (2012), https://www.ea.tuwien.ac.at/fileadmin/t/ea/projekte/ ADRES_Concept/PublizierbarerEndberichtADRES_815674. pdf.

[17] T. Tjaden, J. Bergner, J. Weniger, and V. Quaschning, Representative electrical load profiles of residential buildings in Germany with a temporal resolution of one second, ResearchGate: Berlin, Germany (2015), doi: 10.13140/RG.2.1.3713.1606.

[18] P. Milan, M. Wächter, and J. Peinke, Turbulent Character of Wind Energy, Phys. Rev. Lett. 110, 138701 (2013).

[19] M. Anvari et al., Short term fluctuations of wind and solar power systems, New J. Phys. 18, 063027 (2016).

[20] C. Zhao and Y. Guan, Unified stochastic and robust unit commitment, IEEE Trans. Power Syst. 28, 3353 (2013).

[21] M. Anghel, K. A. Werley, and A. E. Motter, Stochastic model for power grid dynamics, in System Sciences, 2007. HICSS 2007. 40th Annual Hawaii International Conference on (IEEE, New York, 2007), p. 113.

[22] B. Schäfer, M. Matthiae, X. Zhang, M. Rohden, M. Timme, and D. Witthaut, Escape routes, weak links, and desynchronization 
in fluctuation-driven networks, Phys. Rev. E 95, 060203(R) (2017).

[23] B. Schäfer, C. Beck, K. Aihara, D. Witthaut, and M. Timme, Non-Gaussian power grid frequency fluctuations characterized by Lévy-stable laws and superstatistics, Nat. Energy 3, 119 (2018).

[24] H. Haehne, J. Schottler, M. Waechter, J. Peinke, and O. Kamps, The footprint of atmospheric turbulence in power grid frequency measurements, Europhys. Lett. 121, 30001 (2018).

[25] M. F. Wolff et al., Heterogeneities in electricity grids strongly enhance non-Gaussian features of frequency fluctuations under stochastic power input, Chaos 29, 103149 (2019).

[26] K. Schmietendorf, O. Kamps, M. Wolff, P. G. Lind, P. Maass, and J. Peinke, Bridging between load-flow and Kuramoto-like power grid models: A flexible approach to integrating electrical storage units, Chaos 29, 103151 (2019).

[27] B. Schäfer, M. Timme, and D. Witthaut, Isolating the impact of trading on grid frequency fluctuations, in 2018 IEEE PES Innovative Smart Grid Technologies Conference Europe (ISGTEurope) (IEEE, New York, 2018), pp. 1-5.

[28] Z. Li, O. Samuelsson, and R. Garcia-Valle, Frequency deviations and generation scheduling in the nordic system, in PowerTech, 2011 IEEE Trondheim (IEEE, New York, 2011), pp. 1-6.

[29] National Grid, Frequency data (2014-2018), http://www2. nationalgrid.com/Enhanced-Frequency-Response.aspx.

[30] Réseau de Transport d'Électricité (RTE), Network frequency (2014-2019), https://clients.rte-france.com/lang/an/visiteurs/ vie/vie_frequence.jsp.

[31] TransnetBW GmbH, Regelenergie Bedarf + Abruf (2019), https://www.transnetbw.de/de/strommarkt/systemdienstleistun gen/regelenergie-bedarf-und-abruf.

[32] B. Schäfer, D. Witthaut, M. Timme, and V. Latora, Dynamically induced cascading failures in supply networks, Nat. Commun. 9, 1975 (2018).

[33] K. Kashima, H. Aoyama, and Y. Ohta, Modeling and linearization of systems under heavy-tailed stochastic noise with application to renewable energy assessment, in 2015 54th IEEE Conference on Decision and Control (CDC) (IEEE, New York, 2015), pp. 1852-1857.

[34] G. Samorodnitsky and M. S. Taqqu, Stable Non-Gaussian Random Processes. Stochastic Models with Infinite Variance (Chapman and Hall, New York, 1994).

[35] P. H. Westfall, Kurtosis as peakedness, 1905-2014 R.I.P., Am. Stat. 68, 191 (2014).

[36] C. Gardiner, Handbook of Stochastic Methods: for Physics, Chemistry and the Natural Sciences (Springer, Berlin, 1985).

[37] ENTSO-E, Generation Forecast-Day ahead, ENTSO-E https://transparency.entsoe.eu/generation/r2/dayAheadAggrega tedGeneration/show (2019).

[38] H. Kantz and T. Schreiber, Nonlinear Time Series Analysis (Cambridge University Press, Cambridge, 1997).

[39] J. Theiler, S. Eubank, A. Longtin, B. Galdrikian, and J. D. Farmer, Testing for nonlinearity in time series: The method of surrogate data, Physica D 58, 77 (1992).

[40] G. Filatrella, A. H. Nielsen, and N. F. Pedersen, Analysis of a power grid using a Kuramoto-like model, Eur. Phys. J. B 61, 485 (2008).

[41] M. Rohden, A. Sorge, M. Timme, and D. Witthaut, SelfOrganized Synchronization in Decentralized Power Grids, Phys. Rev. Lett. 109, 064101 (2012).

[42] A. Ulbig, T. S. Borsche, and G. Andersson, Impact of low rotational inertia on power system stability and operation, IFAC Proc. 47, 7290 (2014).

[43] X. Zhang, S. Hallerberg, M. Matthiae, D. Witthaut, and M. Timme, Fluctuation-induced distributed resonances in oscillatory networks, Sci. Adv. 5, eaav1027 (2019).

[44] H. Haehne, K. Schmietendorf, S. Tamrakar, J. Peinke, and S. Kettemann, Propagation of wind-power-induced fluctuations in power grids, Phys. Rev. E 99, 050301(R) (2019).

[45] P. Kundur et al., Definition and classification of power system stability IEEE/CIGRE joint task force on stability terms and definitions, IEEE Trans. Power Syst. 19, 1387 (2004).

[46] E. B. T. Tchuisseu et al., Curing Braess' paradox by secondary control in power grids, New J. Phys. 20, 083005 (2018). 